\title{
Salt Body Flooding Using Activation Functions From Machine Learning
}

\author{
Abdullah Alali, Bingbing Sun, Vladimir Kazei, Tariq Alkalifah
}

December 22, 2020

\begin{abstract}
In salt affected regions, conventional full-waveform inversion (FWI) is doomed to fail if there is no prior information of the salt body. Recent studies suggested to regularize the inversion by implementing an automatic flooding using total variation (TV) and Hinge loss functions. We generlize this approach and introduce a family of functions known as "activation functions" in the machine learning discipline that can be used to implement automatic flooding in similar way. In particular, we investigate the automatic flooding using a sigmoid, tanh and exponential linear unit (Elu) functions and apply them for salt body reconstruction on the BP model and report their performance.
\end{abstract}




\section{Introduction}

Full-waveform inversion (FWI) (Tarantola, 1984) is an ill-posed non-linear optimization problem that aims to retrieve the unknown subsurface parameters. Starting from an initial model, FWI updates the model parameters iteratively by minimizing a data misfit until it converges to hopefully global minimum. However, the absence of a good starting model and low frequencies of the data can trap FWI in a local minimum attributed to the well-known cycle-skipping problem (Sun and Alkhalifah, 2019a).

In the presence of salt bodies, the challenges facing FWI are amplified. This is because the salt body has a very high velocity relative to its surrounding media and often we lack of prior knowledge of its existence or structure to include it in the initial model. Conventional industry practice with salt regions is to apply the migrate-pick-flood flow, which is time consuming and prone to error as it requires human interpretation and decision making (Dellinger et al., 2017). For more automatic inversion with less human intervention: Ovcharenko et al. (2018) used a variance-based interpolation to process the conventional FWI updates; Sun and Alkhalifah (2019b) proposed to use more robust objective functions such as the optimal-transport when inverting for the salt. Other studies suggested to apply an automatic flooding by constraining the inversion using a Hing loss (Esser et al., 2016) or regularizing it as a post-inversion process (Kalita et al., 2019). In this abstract, we focus on the automatic flooding where the velocity of the top of the salt is smeared down in depth. The top of the salt is captured by the FWI and is followed by a large velocity drop. A flooding regularization identifies this drop and penalize it to mimic the conventional pick-flood flow automatically.

A family of functions in the machine learning discipline known as "activation functions" are widely used in classification (Nwankpa et al., 2018). Here, we show that these functions can be used to identify the large velocity drop and flood it. In fact, the hing loss that Esser et al. (2016) used, belongs to this family where it is known as the rectified linear unit (ReLu). Among the family of activation functions, we will investigate the sigmoid, tanh and exponential linear unit (Elu) functions. They behave similarly but differ in the range. We validate the approach by inverting for the BP 2004 model.

\section{Theory}

We follow Kalita et al. (2019) approach where we alternate between two optimization problems until we converge. The first problem is an FWI with TV regularization and the second one is the automatic flooding.

The objective function for the FWI+TV has the form:

$$
J_{F W I+T V}(m)=\left\|d^{m}-d^{o b s}\right\|_{2}^{2}+\lambda_{T V}\|\nabla m\|,
$$

where $d^{m}$ and $d^{o b s}$ are the modeled and the observed data, respectively. $\lambda_{T V}$ is a regularization coefficient for the TV regularization, which reads,

$$
\|\nabla m\|=\sqrt{\left(\frac{\partial m}{\partial x}\right)^{2}+\left(\frac{\partial m}{\partial z}\right)^{2}+\varepsilon .}
$$

$\varepsilon$ is a smoothing parameter to stabilize the gradient when $\nabla m=0$. The flooding is then applied to the inverted model by minimizing the following objective,

$$
J_{F}(m)=\left\|\left(m-m^{\prime}\right)\right\|_{2}^{2}+\lambda_{F}\|\mathscr{F}(m)\|
$$

where $m^{\prime}$ is the output of the first optimization (equation 1). $\mathscr{F}(m)$ is a penalty function that penalizes the drop in velocity by flooding the region with higher velocity and $\lambda_{F}$ is a coefficient controlling the trade-off between the two norms.

The velocity drop is quantified by the negative values of the vertical derivative of the model $m_{z}$. A 
good choice of $\mathscr{F}(m)$ is a function that gives a high weight when the negative of $m_{z}$ is large. Many activation functions have this property embedded in them such as the sigmoid $(\sigma), \tanh (\Gamma)$ and Elu $(\mathscr{E})$ given, respectively, by:

$$
\sigma(x)=\frac{1}{1+e^{-x}} ; \quad \Gamma(x)=\frac{e^{x}-e^{-x}}{e^{x}+e^{-x}} ; \quad \mathscr{E}^{*}(x)=\left\{\begin{array}{cc}
x^{p} & x>0 \\
\alpha\left(e^{x}-1\right) & x<=0
\end{array} .\right.
$$

The star in the Elu is to indicate that we have slightly modified the function by introducing a power $(p)$ to make the function more sensitive toward the larger velocity drops. To properly use these functions in implementing the flooding, the argument should be $-m_{z}$ rather than $m_{z}$ as we want to penlize the gradient with he negative value. Figure 1 shows the sigmoid, tanh and Elu, respectively. For the purpose of penalizing only the velocity drop, we only consider using the functions when $m_{z}$ is negative (the dashed region in Figure 1).

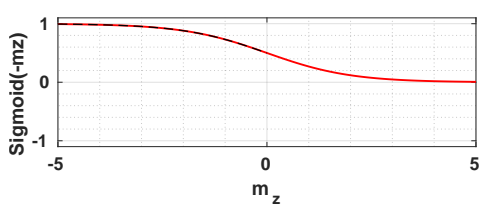

(a)

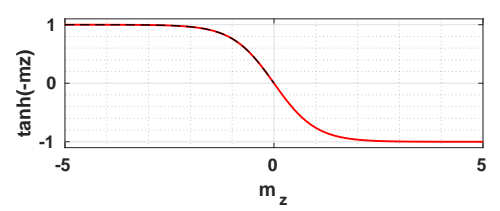

(b)

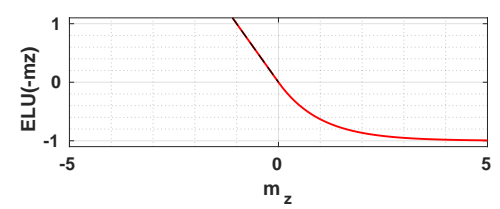

(c)

Figure 1 Activation functions where $(a)$ is the sigmoid, $(b)$ is the tanh and $(c)$ is the Elu function.

The gradient for $\|\mathscr{F}(m)\|$ can be obtained as,

$$
\frac{\partial \mathscr{F}(m)}{\partial m}=-\frac{\partial}{\partial z} \frac{\partial \mathscr{F}}{\partial m_{z}} .
$$

For the sigmoid, the gradient is,

$$
\frac{\partial \sigma\left(-m_{z}\right)}{\partial m}=\frac{\partial}{\partial z} \frac{e^{m_{z}}}{\left(1+e^{m_{z}}\right)^{2}}=e^{m_{z}} \frac{1-e^{m_{z}}}{\left(1+e^{m z}\right)^{3}} m_{z z},
$$

where $m_{z z}$ is the second order vertical derivative. The gradient for the tanh reads,

$$
\frac{\partial \Gamma\left(-m_{z}\right)}{\partial m}=\frac{\partial}{\partial z}\left(1-\tanh \left(m_{z}\right)^{2}\right)=-2 \tanh \left(m_{z}\right)\left(1-\tanh \left(m_{z}\right)^{2}\right),
$$

and for the modified Elu, the gradient is,

$$
\frac{\partial \mathscr{E}\left(-m_{z}\right)}{\partial m}=\frac{\partial}{\partial z} p(-m z)^{p-1}=p(p-1)\left(-m_{z}\right)^{p} m_{z z} .
$$

Figure 2 shows a 1D velocity and the corresponding updates (i.e. negative of the gradient) for the three functions. When there is a drop in the velocity, the update has positive and negative directions (Figure 2(a)) that are controlled by the sign of $m_{z z}$. For the purpose of the flooding, we only consider the positive update as shown in Figure 2(b). Note how the functions honor the largest drop by giving it the highest update while almost neglecting the small ones at the same time.

\section{Numerical Example}

We test the flooding functions on the west part of the BP 2004 velocity model given in Figure 3(a). The constant density acoustic constant wave equation is used to generate the data. We used 248 sources at intervals of $60 \mathrm{~m}$ and at depth of $2 \mathrm{~m}$ with a Ricker wavelet of peak frequency of $5.5 \mathrm{~Hz}$ and $3 \mathrm{~Hz}$ minimum frequency. The data are recorded by 801 receivers at a depth of $2 \mathrm{~m}$ as well. We perform frequency continuation in the inversion where initially low frequencies are used in the inversion and progressively we include the higher frequencies until all frequencies in the bandwidth of the data are included. We 


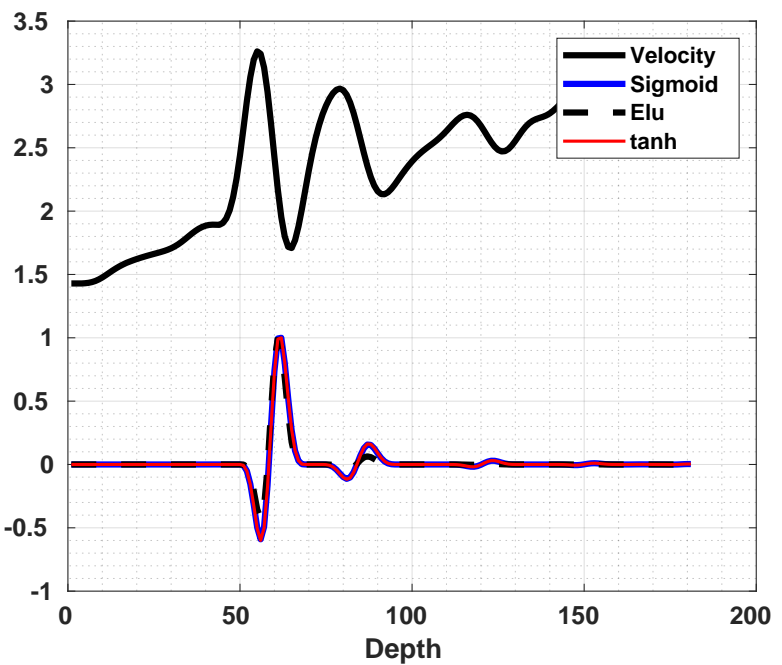

(a)

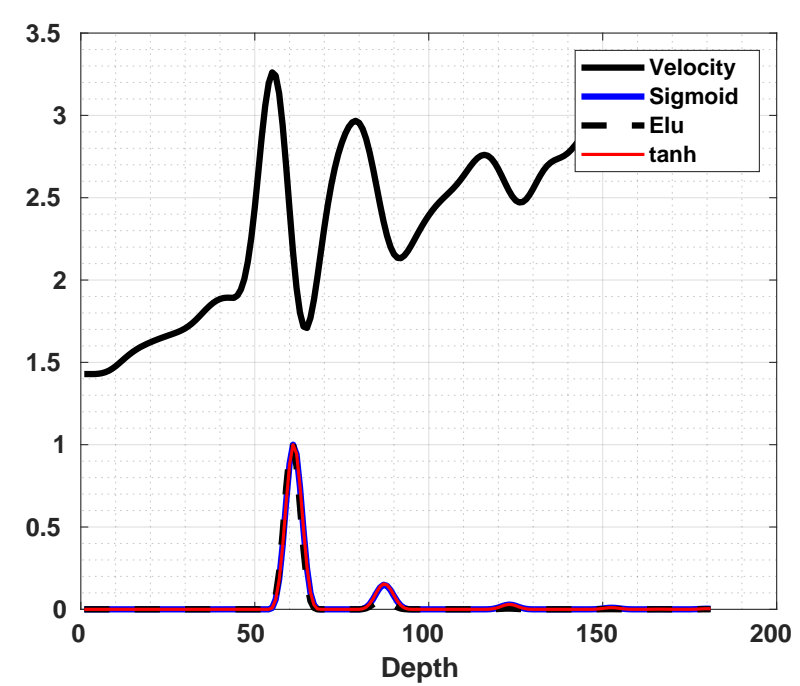

(b)

Figure $21 D$ velocity profile with the corresponding updates for the sigmoid, tanh and elu. (a) the positive and negative update and (b) the positive update only.

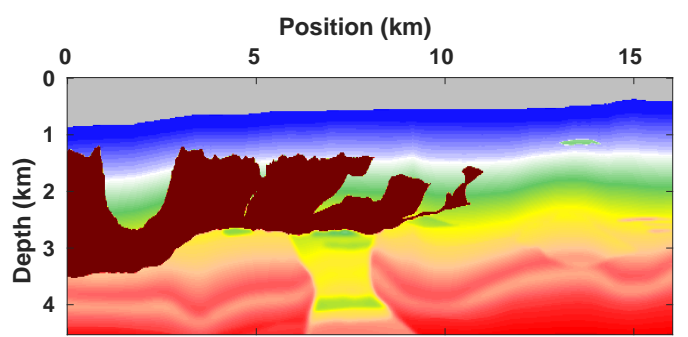

(a)

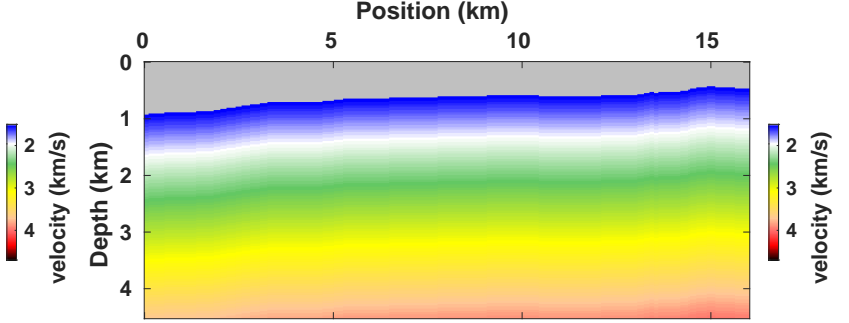

(b)

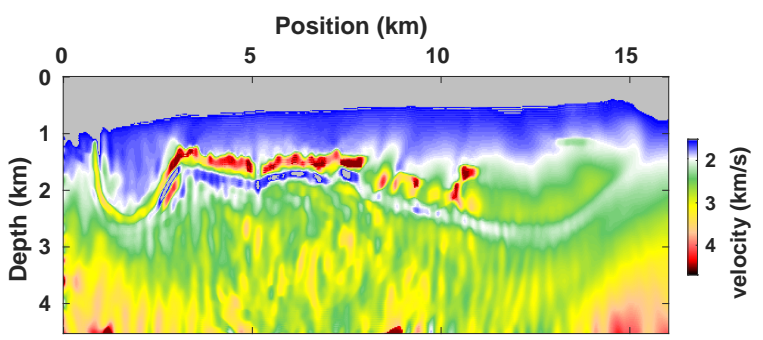

(c)

Figure 3 (a) BP 2004 model down scale by half, (b) inital mode and (c) the inversion result for FWI+TV

start the inversion with $3.5 \mathrm{~Hz}$ followed by $4.0 \mathrm{~Hz}, 6.0 \mathrm{~Hz}$ and then the full bandwidth. The initial model used for inversion is shown in Figure 3(b) with a linearly increasing velocity in depth starting from the water bottom. The first FWI+TV is shown in Figure3(c). We can see how the model capture the top of the salt and has a large drop beneath it.

The results of applying the flooding on the first inversion result is shown in the first column in Figure 4 using the sigmoid, tanh and Elu, respectively. Simoid and tanh flooding results are similar while the elu is slightly different. We repeat the cycle of FWI-Flooding until we reach convergence. The final inverted models are shown in the second column in Figure 4. We can see that the inversion recovers the salt shape fairly well. We will share details of the performance of the different flooding function in the presentaion of the work.

\section{Conclusion}

We generalize the approach of TV and Hing loss regularization for salt body inversion; we utilized the activation functions used in machine learning discipline in flooding regularization. In particular, we 


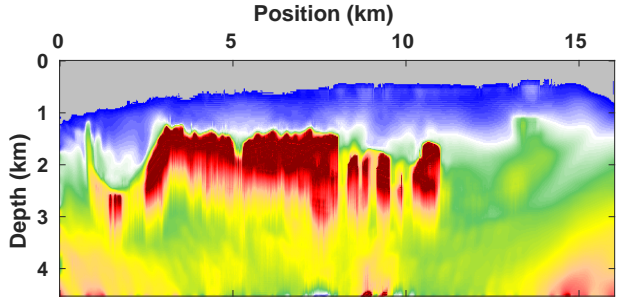

(a)

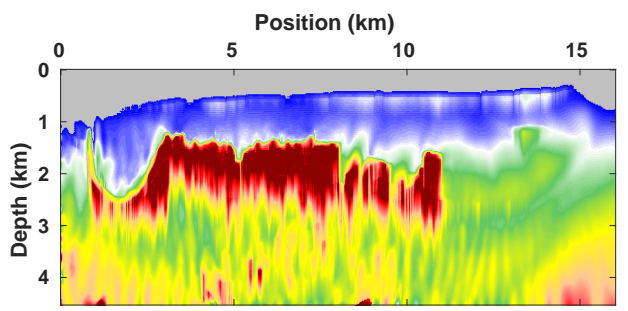

(c)

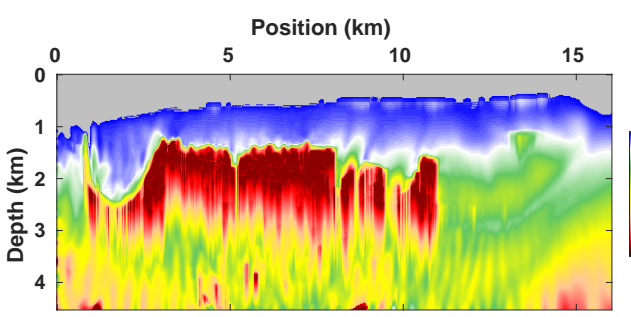

(e)

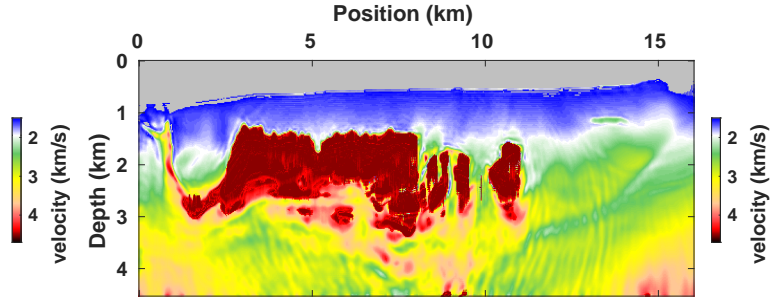

(b)

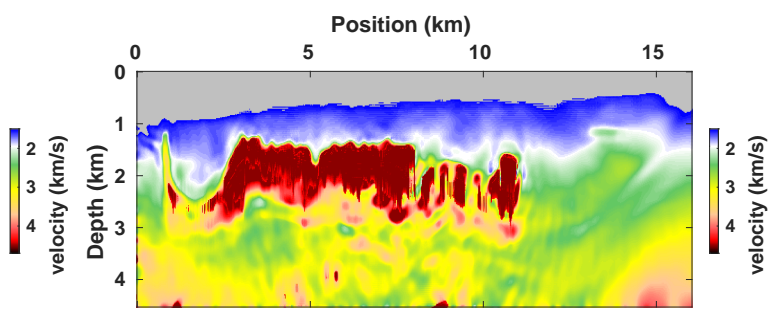

(d)

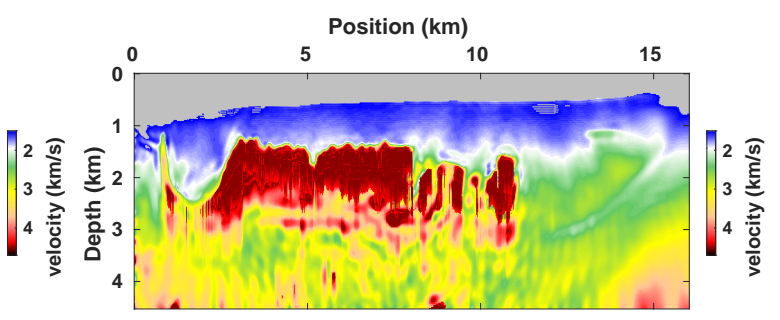

(f)

Figure 4 First column (a,c,e) are the flooding of the inverseion result in Figure 3(c) using the sgmoid, tanh and Elu, respectively. The second column $(b, d, f)$ is the correspondence FWI inversion after a few FWI-flooding cycles.

tested the sigmoid, tanh and Elu on the BP 2004 model and achieved good flooding results.

\section{Acknowledgment}

We would like to thank King Abdullah University of Science \& Technology (KAUST) for its support. We also thank the Sismic Wave Analysis Group for the fruitful discussion especially Mahesh Kalita.

\section{References}

Dellinger, J., Brenders, A.J., Sandschaper, J., Regone, C., Etgen, J., Ahmed, I. and Lee, K. [2017] The garden banks model experience. The Leading Edge, 36(2), 151-158.

Esser, E., Guasch, L., Herrmann, F.J. and Warner, M. [2016] Constrained waveform inversion for automatic salt flooding. The Leading Edge, 35(3), 235-239.

Kalita, M., Kazei, V., Choi, Y. and Alkhalifah, T. [2019] Regularized full-waveform inversion with automated salt flooding. Geophysics, 84(4), R569-R582.

Nwankpa, C., Ijomah, W., Gachagan, A. and Marshall, S. [2018] Activation functions: Comparison of trends in practice and research for deep learning. arXiv preprint arXiv:1811.03378.

Ovcharenko, O., Kazei, V., Peter, D. and Alkhalifah, T. [2018] Variance-based model interpolation for improved full-waveform inversion in the presence of salt bodies. Geophysics, 83(5), R541-R551.

Sun, B. and Alkhalifah, T. [2019a] The application of an optimal transport to a preconditioned data matching function for robust waveform inversion. Geophysics, 84, no. 6, R923-R945.

Sun, B. and Alkhalifah, T. [2019b] Salt body inversion using an optimal transport of the preconditioned matching filter. In: 81st EAGE Conference and Exhibition 2019, 2019. European Association of Geoscientists \& Engineers, 1-5.

Tarantola, A. [1984] Inversion of seismic reflection data in the acoustic approximation. Geophysics, 49(8), 1259-1266. 